\title{
La question du monopole d'émission de la monnaie : le débat banque centrale contre banque libre chez les économistes français (1860-1875)
}

Jean-Paul Domin

\section{OpenEdition}

\section{Journals}

Édition électronique

URL : http://journals.openedition.org/ress/231

DOI : $10.4000 /$ ress.231

ISSN : 1663-4446

Éditeur

Librairie Droz

\section{Édition imprimée}

Date de publication : 1 juillet 2007

Pagination : 185-202

ISBN : 978-2-600-01155-6

ISSN : 0048-8046

Référence électronique

Jean-Paul Domin, «La question du monopole d'émission de la monnaie : le débat banque centrale contre banque libre chez les économistes français (1860-1875) », Revue européenne des sciences sociales [En ligne], XLV-137 | 2007, mis en ligne le 01 juillet 2010, consulté le 19 avril 2019. URL : http:// journals.openedition.org/ress/231; DOI : 10.4000/ress.231 


\section{LA QUESTION DU MONOPOLE D'ÉMISSION DE LA MONNAIE: Le débat banque centrale contre banque libre chez les économistes français (1860-1875)}

La question de l'organisation du système bancaire et des conditions d'émission de la monnaie sont toujours d'actualité. En effet, la dislocation des anciennes démocraties populaires a provoqué des phénomènes de désorganisation des échanges et a favorisé l'émergence de dysfonctionnements monétaires importants. Le retour du troc et la volonté de certains de le contourner ont conduit à la formation de structures bancaires alternatives refusant toutes formes de réglementation et de supervision par une autorité. Cette grave crise a favorisé la hausse de l'inflation et a repoussé les limites de la création monétaire ${ }^{1}$.

L'existence même d'une banque centrale est une question fondamentale. Deux modèles s'opposent à ce sujet : le free banking et le central banking. Le premier propose une mise en concurrence radicale des banques sans contrôle, ni banque centrale et s'appuie sur une seule règle : la définition d'une unité de compte commune. Ce modèle correspond essentiellement à un système de paiement où une monnaie bancaire est convertible en monnaie marchandise. Le central Banking repose sur un modèle hiérarchique dans lequel les monnaies sont unifiées par une contrainte de conversion. Ce système de paiement constitue une forme moderne d'organisation monétaire où l'unité de compte et les moyens de paiement sont liés. Ces derniers sont convertibles en monnaie fiduciaire. Finalement, la régulation monétaire dans le modèle du central banking résulte d'une politique économique alors que dans le free banking, elle est régie par un mécanisme de marché.

Cette opposition de principe va faire l'objet d'un débat chez les économistes sous le Second Empire La période qui s'étend de 1850 à 1860 est marquée par l'apogée de la prospérité agricole, l'élaboration du système bancaire, le développement des grands travaux et la naissance du réseau ferroviaire ${ }^{2}$. Cet essor s'appuie essentiellement sur les établissements de crédit. La Banque de France, par crainte de la concurrence, leur refuse l'appellation de banques qui lui est réservée. Cependant, des structures nouvelles vont progressivement se mettre en place pour faciliter le financement de l'industrie : le Crédit Mobilier (1852), le Crédit Foncier (1852), le Crédit Lyonnais (1863) et la Société Générale (1864). Ces structures se spécialisent dans le crédit commercial et le financement des projets importants. Ces banques commerciales ne peuvent obtenir un droit d'émission qui, en droit français, revient à la Banque de France.

\footnotetext{
Aglietta (1992), pp. 675-698.

Asselain (1984), p. 146.
} 
Ce travail propose une synthèse des débats théoriques sur la question du monopole d'émission de la Banque de France sous le Second Empire et une interprétation de la logique économique sous-jacente. Après avoir replacé le débat banque centrale contre banque libre dans sa perspective historique (I), nous montrerons que celui-ci est marqué par une richesse théorique et s'inscrit, plus généralement, dans la problématique de l'intervention de l'Etat (II).

\section{DÉBATS JURIDIQUES AUTOUR DE LA QUESTION DU MONOPOLE}

Le débat sur la structure de l'émission monétaire est assez ancien en France. L'apparition de la Banque de Savoie dans le système monétaire va le relancer.

\section{La banque de Savoie et la question du monopole de la Banque de France}

De nombreuses études ont montré que le système de banque centrale apparaît après des périodes de troubles monétaires importants. Ainsi, l'élaboration d'un modèle monétaire de central banking ne proviendrait pas de l'instabilité d'un système privé et concurrentiel, mais plutôt d'une volonté politique de capturer le pouvoir monétaire. Le système existant sous le Second Empire est le fruit d'une longue évolution.

\section{De la Banque libre à la banque centrale}

Pour comprendre les raisons de la mise en œuvre d'un monopole d'émission, il faut revenir à la Révolution française. La situation financière en 1789 est assez défavorable. Pour tenter de l'améliorer, l'Assemblée constituante décide, le 2 novembre 1789, de confisquer les biens du clergé et de les mettre à la disposition de la Nation. Le 19 décembre, elle crée les assignats, définis comme des « certificats de monnayage du sol » et représentant une créance de l'Etat portant un intérêt de $5 \%$, remboursable en biens du clergé.

Cette monnaie pose un double problème : elle est émise en très grande quantité, ce qui favorise l'apparition d'une inflation et représente une valeur gagée sur les biens ecclésiastiques confisqués et totalement non liquides ${ }^{3}$. Le système monétaire est complètement désorganisé et de nombreuses communes créent des caisses patriotiques dont la fonction est d'échanger des assignats contre des billets de confiance (papier-monnaie d'un plus petit montant). Les pouvoirs publics vont progressivement contrôler cette organisation libre ${ }^{4}$ : le 8 novembre 1792, l'Assemblée nationale interdit la création d'établissement émetteur de monnaie 5 .

De 1796 à 1803, le pays va connaître une courte période de free banking. Dès 1796, le décret interdisant la création de banques émettrices est annulé. Le 29 juin 1796, un groupe d'hommes d'affaires crée la Caisse des comptes courants.

\footnotetext{
Aftalion (1987).

4 White (1987), pp. 251-276.

5 Aubin (1991), pp. 745-762.
} 
Cet établissement au capital de cinq millions de francs émet pour un montant de vingt millions de billets remboursables et escompte des lettres de change. Progressivement, le dispositif va se structurer autour de quelques banques : la Caisse d'escompte du commerce (créée le 4 novembre 1797 et qui émet pour vingt millions de francs de billets), la Société générale du commerce de Rouen (fondée le 20 avril 1798 et émettant pour vingt-et-un millions de francs de billets) et le Comptoir commercial (appelée aussi Caisse Jabach et créé courant 1800). Cette évolution est couronnée, le 18 janvier 1800, par la création de la Banque de France. Cet établissement est ouvert avec un capital de trente-six millions de francs issus de l'ancienne Caisse des comptes courants. En 1802, la Banque de France absorbe la Caisse d'escompte du commerce.

Cette période de pluralité cesse avec la loi du 14 avril 1803 qui donne le monopole d'émission à la Banque de France et qui interdit les banques de province sauf avec une dérogation du gouvernement. La loi du 22 avril 1806 proroge le monopole d'émission pour vingt-trois ans supplémentaires et modifie les statuts de la Banque. Les dirigeants sont désormais nommés par le gouvernement et non plus par les administrateurs et leur nombre diminue de dix-huit à trois. La législation du 18 mai 1808 l'autorise à créer des filiales dans les villes de province. Des succursales sont ouvertes à Rouen, Lyon et Lille, elles ont un monopole local d'émission des billets.

La Restauration tente de libéraliser le système en autorisant la création de banques privées au droit d'émission limité. Ces banques dites départementales ont des capacités d'émission limitées au triple de leurs réserves d'or, mais leur nombre augmente sans cesse: six établissements sont constitués entre 1835 et 1838. Face à cette concurrence, la Banque de France réagit en créant quinze filiales. En 1840, elle demande au gouvernement de ne plus autoriser la création de nouveaux établissements. Cette décision va entraîner la diminution du nombre des banques départementales.

La crise politique de 1848 déclenche une panique bancaire ${ }^{6}$. Le gouvernement cherche à protéger les réserves monétaires de la Banque de France et déclare le cours forcé du billet de banque sur l'ensemble du territoire. Le 27 avril 1848, les pouvoirs publics ordonnent la fusion des succursales départementales à la Banque de France, qui donne à cette dernière un monopole d'émission de fait. Enfin, la loi du 9 juin 1857 renouvelle son privilège pour une durée de quarante ans, libéralise la fixation du taux de l'escompte et permet à la banque de conserver les bénéfices acquis supérieurs d'un intérêt de $6 \%$.

\section{La contestation effective du monopole d'émission}

Le rattachement de Nice et de la Savoie à la France, prévu par le Traité de Turin du 24 mars 1860 va modifier la situation. La Convention franco-sarde du 23 août 1860 prévoit expressément que : «La Banque établie à Annecy continuera à jouir dans la Savoie des droits et privilèges... ». Pour les délégués de la Banque de Savoie, l'exercice de cette dernière n'est donc pas limité au territoire dans lequel elle est née. L'établissement ne peut donc pas être assimilé à une simple

6 Cameron (1967). 
banque départementale ${ }^{7}$. Pour le jurisconsulte Dufaure, interrogé par les conseillers savoyards, l'existence d'un privilège d'émission pour la Banque de Savoie est légale. Cette position est confirmée par la Cour de Cassation le 7 juillet 1862. Le privilège de la Banque de France est impuissant devant celui de la Banque de Savoie, puisque ce dernier est antérieur au Traité de Turin. D'autre part, n'étant pas rétroactif, il ne porte donc aucune atteinte aux droits privés antérieurement acquis et ne peut donc s'appliquer aux territoires étrangers ${ }^{8}$. La concurrence entre les deux banques est donc juridiquement possible.

Les délégués de la Banque de Savoie proposent le 31 octobre 1860, après une entrevue avec Napoléon III, une fusion au pair de leurs actions avec celles de la Banque de France. Mais le Conseil de régence refuse cette fusion le 15 février 1861. Celui-ci, en échange, entend racheter le privilège d'émission de la première pour un montant de 600000 francs. Après avoir refusé cette proposition et constatant qu'aucune entente n'est possible, les conseillers savoyards renoncent à un arrangement avec la Banque de France et opèrent un rapprochement le 22 septembre 1861 avec les frères Péreire9.

Isaac et Emile Péreire ont intérêt à encourager la pluralité d'émission afin de disposer de moyens financiers plus importants. La possibilité qui leur est donnée de procéder à l'émission de monnaie fiduciaire constitue alors une solution intéressante pour le Crédit mobilier qui est à la recherche de ressources nouvelles. L'occasion leur est d'autant plus favorable que le droit semble être pour eux. Les délégués de la Banque de Savoie leur accordent, le 30 mars 1862, une autorisation facultative pour réorganiser cette institution. Des prolongations leurs sont accordées le 20 novembre 1862 jusqu'au 1er février 1863, puis le 26 mars jusqu'au 26 septembre.

Finalement, les frères Péreire proposent une alliance stratégique à la Banque de Savoie. La convention du 18 septembre 1863 lui confie la gestion des six compagnies de chemins de fer, l'autorise à émettre leurs bons et obligations sur autorisation du gouvernement. Elle permet à la Banque de gérer les fonds disponibles des compagnies. En échange, le Crédit Mobilier injecte trente-six millions de francs dans la Banque. La convention prévoit enfin que le gouverneur et le sousgouverneur de la banque de Savoie seront nommés par le gouvernement. L'objectif est de faire de cet établissement la banque de toutes les compagnies de chemin de fer.

Face à ce que la Banque de France considère comme une provocation, les pouvoirs publics ne tardent pas à réagir. Le gouvernement décide la mise sous séquestre des biens de la Banque de Savoie au motif qu'elle a violé ses statuts ${ }^{10}$.

${ }^{7}$ Note des délégués de la Banque de Savoie à son excellence M. le ministre des Finances de France, Paris, éditions Dentu, 1861.

${ }^{8}$ La Banque de France et la Banque de Savoie devant l'intérêt public, Paris, éditions Paul Dupont, 1863.

${ }^{9}$ Ces derniers constituent, au sein de l'Empire, un groupe financier considérable et ont réussi à tisser des liens avec de nombreuses familles européennes : les Oppenheim de Cologne, les Heine de Hambourg, les Baring de Londres. Autin (1984). Leur volonté de constituer un pôle financier important autour de la Banque de Savoie s'inscrit dans le combat qu'ils mènent contre James de Rothschild. Bouvier (1992).

${ }^{10}$ Bulletin des lois, $\mathrm{n}^{\circ} 1256,24$ octobre 1864. 
Deux décrets du 8 avril 1865 édictent la suppression du privilège d'émettre de la Banque de Savoie et autorisent la Banque de France à créer une nouvelle succursale. Malgré cette décision politique et sous l'impulsion des frères Péreire, le débat sur le monopole d'émission va se développer dans les milieux économiques et financiers.

\section{Les premiers débats autour du monopole d'émission}

Cette affaire politico-financière va favoriser la résurgence du débat sur la question du monopole d'émission ${ }^{11}$. Bon nombre de théoriciens libéraux vont demander sa suppression pour des raisons économiques. Face à eux, les partisans du monopole vont axer leur argumentation sur les questions de sécurité et d'unité du signe monétaire.

\section{La contestation économique du monopole}

Progressivement et parallèlement à l'affaire juridique entre la Banque de France et la Banque de Savoie, certains économistes vont contester la politique monétaire et réclamer la suppression du monopole d'émission. Leur argumentation porte avant tout sur la politique monétaire de la Banque de France qui est, selon eux, entachée de fautes graves. En effet, celle-ci diminue fortement le volume de la masse monétaire et augmente le taux de l'escompte. La réduction de l'encaisse métallique résulte de la transformation d'une grande partie du capital en rentes sur l'Etat. La critique est double. Les pouvoirs publics et les dirigeants de la Banque de France privilégient le développement des rentes sur l'Etat et cherchent à accroître les profits de l'établissement. La politique monétaire est d'autant plus absurde qu'elle favorise l'augmentation des dividendes des actionnaires au détriment de l'activité économique et industrielle du pays. En effet, l'élévation du taux de l'escompte limite les investissements des industriels et nuit plus généralement au développement économique ${ }^{12}$.

Ce point de vue est contestable. En effet, de 1845 à 1870, la masse monétaire disponible a plus que doublé passant de 3900 à 8600 millions de francs. Mais le gonflement de la masse monétaire est accompagné par une transformation plus importante de sa structure. Ainsi, l'augmentation de la circulation fiduciaire met en évidence la multiplication des échanges. Alain Plessis estime que cette évolution correspond plus à une véritable révolution, marquant la fin des pénuries monétaires ${ }^{13}$.

La contestation du principe du monopole d'émission doit conduire tout naturellement à l'émergence d'arguments favorables au libéralisme bancaire. La pétition de Théophile Furet s'inscrit pleinement dans cette perspective. Le monopole d'émission de la Banque de France favorise, selon son auteur, l'augmentation du prix des services bancaires. Il semble donc logique d'en limiter le développe-

11 Au $1^{\text {er }}$ mars 1865,25 auteurs ont publié 30 brochures sur cette question. Parmi celles-ci : Reyre (1864), Dagneaux (1864), Bonnet (1864), Valdenaire (1864).

${ }^{12}$ La Banque de France et la Banque de Savoie devant l'intérêt public, Paris, Imprimerie Paul Dupont, 1863.

13 Plessis (1976), p. 92. 
ment ${ }^{14}$. Pour lui, les banques n'ont pas été créées pour faire office de maisons d'escomptes, mais plutôt pour favoriser l'essor du crédit. Il apparaît plus intéressant d'instituer une forme nouvelle de pluralité d'émission assise sur une concurrence qui permette de favoriser la circulation monétaire et la baisse du taux de l'escompte. L'idée d'une concurrence bancaire est lancée. Le débat soulevé par les frères Péreire et par Théophile Furet va prendre de l'ampleur lors de la discussion de la pétition au Sénat. Comme le rappelle Michel Chevalier, celle-ci «soulève une question qui est d'intérêt public au premier chef, celle de l'organisation du crédit en France $»^{15}$.

Pour les partisans du libéralisme, l'apparition de la Banque de Savoie dans le système bancaire est salutaire et constitue un progrès évident. Certains auteurs parlent même d'une vulgarisation du crédit de manière à "faire descendre son influence bienfaisante jusque dans les régions les plus infimes du corps social $»^{16}$. Malgré la publication de nombreuses brochures sur cette question, l'argumentation se structure essentiellement autour d'une critique du monopole et non des bienfaits de la concurrence. En revanche, pour les partisans du monopole, les arguments favorables à la pluralité bancaire sont largement discutables.

\section{Le monopole d'émission, élément de croissance et de sécurité}

Le rapport de Monsieur Hubert-Delisle est significatif de l'émergence d'une nouvelle logique. La concurrence ne peut absolument pas être remise en cause puisque c'est elle «qui donne une vive et bienfaisante impulsion à la production qui conduit aux plus sérieuses améliorations... ${ }^{17}$. Mais dans le domaine de la monnaie, la concurrence n'est pas souhaitable dans la mesure où elle remettrait en cause l'équilibre du système. L'insécurité bancaire est un risque important. L'auteur, en s'appuyant sur l'argumentation de Robert Peel, cite les exemples de l'Angleterre $^{18}$ et des Etats-Unis. Pour la première, en trois ans, il y aurait eu 240 faillites et 89 banqueroutes. Aux Etats-Unis, en 1836, 959 banques se sont trouvées dans l'impossibilité de faire face à leurs engagements. Cet argument est

14 Furet (1864).

15 Rapport et discussion d' une politique relative à la Banque de France et à la Banque de Savoie, Séances du Sénat des 20 et 30 mai 1864, Paris, éditions Pankoucke, 1864, p. 120.

${ }^{16}$ La Banque de France et la Banque de Savoie devant l'intérêt public, Paris, Imprimerie Paul Dupont, 1863, p. 18.

${ }^{17}$ Rapport de Monsieur Hubert-Delisle chargé d' examiner une pétition relative à la réduction et à la fixation du taux de l'escompte des banques privilégiées, Sénat, Séance du 20 mai 1864.

${ }^{18}$ Le modèle bancaire anglais évolue entre banque libre et banque centrale. Le Tunnage Act de 1695 crée la Banque d'Angleterre. C'est une banque sous contrat, dotée d'une responsabilité limitée. Dès 1697, celle-ci voit ses privilèges étendus (droit d'émission, monopole de détention des encaisses de l'Etat, etc.). Sa position privilégiée exclut les autres établissements. A la fin du XVIII ${ }^{\mathrm{e}}$ siècle, elle entre dans une période de crise : les prêts accordés sont trop importants par rapport aux ressources financières. Le Parlement vote alors la fin de la convertibilité des billets. La loi de 1812 accorde aux billets de banque de la Banque d'Angleterre le statut de monnaie à cours légal. En 1833, le Joint Stock Act reconnaît aux autres établissements le droit d'émettre. Il faut attendre la réforme de Peel de 1844 pour que la Banque d'Angleterre reçoive effectivement le monopole d'émission. La banque est alors divisée en deux départements. Le marché du crédit est séparé du marché de la monnaie. La loi concrétise la victoire de la currency school. 
récurrent chez les partisans du monopole, il est contesté par les tenants de la concurrence qui estiment que ces chiffres sont exagérés.

La critique de la concurrence est plus vive encore quand l'auteur montre que l'élaboration d'une pluralité d'émission est incohérente. En effet, la concurrence entre les banques est plus un mirage conduisant à des transactions avantageuses, mais souvent irréelles. Les établissements de crédit ont l'impression de créer de la richesse alors qu'ils favorisent l'apparition de dettes. Cette idée s'appuie sur le concept de currency school (école de la circulation) selon lequel l'émission des billets de banque doit être réglée par l'encaisse de l'établissement émetteur. Ainsi, tout billet doit avoir une contrepartie métallique dans l'institut d'émission. Monsieur Hubert-Delisle admet explicitement que la pluralité bancaire favoriserait la baisse du taux de l'escompte, mais il précise que celle-ci se traduirait inéluctablement par un comportement visant à attirer la clientèle.

L'auteur pense donc que le monopole d'émission doit rester une prérogative publique : «le droit d'émettre le papier fiduciaire est un attribut qui échappe à la concurrence, c'est une faculté spéciale, conservatrice, essentielle à l'ordre public... ${ }^{19}$. L'argument de la confiance et de la sécurité est sous-jacent. La confiance dans le billet au porteur et la qualité de la circulation de la monnaie dépendent de la faculté de remboursement. En revanche, l'ouverture du système bancaire à la concurrence favoriserait la confusion et romprait l'unité bancaire ${ }^{20}$.

Lors de la discussion au Sénat, en mai 1864, Michel Chevalier, proche des frères Péreire, soutient la thèse de la pluralité bancaire qui est, selon lui, un bienfait économique. Il prend l'exemple de l'Ecosse ${ }^{21}$ et des Etats-Unis où la liberté d'émission favorise la croissance industrielle. Il montre ainsi que l'élaboration de Joint Stock Banks, distribuant des dividendes à leurs clients, serait une solution favorable et dynamique. Il revient sur la question de la pluralité et montre qu'elle ne remet nullement en cause l'unité du signe monétaire et n'exclut ni l'ordre, ni la réglementation. Le ministre d'Etat, Eugène Rouher, lui rétorque qu'il est indispensable que le système bancaire s'articule autour d'un signe commun ${ }^{22}$.

${ }^{19}$ Rapport de Monsieur Hubert-Delisle chargé d' examiner une pétition relative à la réduction et à la fixation du taux de l'escompte des banques privilégiées, Sénat, Séance du 20 mai 1864, p. 33.

${ }^{20}$ Commez (1864).

${ }^{21}$ Le modèle écossais constitue l'archétype du free banking. La Banque d'Ecosse est créée en 1695 et reçoit immédiatement un monopole d'émission sur un territoire déterminé pour 31 ans. Le contrat est renouvelable en 1726. En 1704, elle instaure une clause d'option qui lui permet de suspendre la convertibilité des billets en échange d'un taux d'intérêt annuel de $5 \%$ pendant la période de suspension. En 1717, Londres, craignant les activités politique de la Banque d'Ecosse favorise la création d'un établissement concurrent ayant le droit d'émettre des billets : La Banque Royale d'Ecosse. En 1728, après une période de troubles politiques, la Banque d'Ecosse suspend sa convertibilité alors que son concurrent la conserve. A partir de 1746, la concurrence entre les deux banques va s'intensifier en raison de l'apparition sur le marché de deux nouvelles structures : la Glasgow Ship Bank et la Glasgow Arms Bank. Cette situation favorise l'élaboration de nouveaux produits bancaires, mais également les ententes. Les deux premières acceptent mutuellement leurs billets et, en 1771, décident de faire de même avec les billets de leurs concurrents. Au XIX ${ }^{\mathrm{e}}$ siècle, le système évolue grâce à la constitution d'un actionnariat important. Dans le même temps, les établissements publient des informations pour faciliter l'unité. En 1845, suite à la modification de la loi anglaise, l'Ecosse adopte le Scottish Bank Act qui donne le monopole d'émission aux banques existantes.

${ }^{22}$ Rapport et discussion d' une politique relative à la Banque de France et à la Banque de Savoie, Séances du Sénat des 20 et 30 mai 1864, Paris, éditions Pankoucke, 1864, p. 177. 
Suite à ce premier débat, le Sénat vote majoritairement pour le maintien du monopole. Cependant Michel Chevalier a émis l'idée de la création d'une commission d'enquête sur le monopole. Celle-ci est relayée par Darimon dans La Presse et par Isaac Péreire. Suite à d'autres pétitions lancées par des commerçants et des négociants et le conseil de Régence de la Banque de France, le 9 janvier 1865, Napoléon III approuve l'idée d'une commission d'enquête et demande au ministre d'Etat Eugène Rouher de la présider. La plupart des économistes européens vont y participer, ce qui fait de cette période « la plus enthousiasmante et la plus productive ${ }^{23}$. La composition du Conseil supérieur du Commerce, de l'Agriculture et de l'Industrie, qui est chargé d'organiser l'enquête, est assez favorable au principe du monopole d'émission de la Banque de France. Parmi ses membres, seul Michel Chevalier soutient les frères Pereire ${ }^{24}$.

\section{L'ENQUÊTE \\ ET L'ACCEPTATION PROGRESSIVE DU MONOPOLE}

Le débat organisé par la Commission d'enquête s'articule autour de deux grandes tendances : la pluralité et le monopole d'émission.

\section{La pluralité d'émission entre concurrence, oligopole et duopole}

Les avis des partisans de la pluralité sont assez partagés. Trois tendances se dégagent nettement dans les débats : la concurrence illimitée, la structure oligopolistique (concurrence limitée entre quelques établissements autorisés) et le duopole.

\section{Le système concurrentiel}

Parmi les partisans de la concurrence, Jean-Gustave Courcelle-Seneuil est vraisemblablement le défenseur le plus inconditionnel de la liberté absolue des services bancaires : "mon opinion est qu'il est bon que la concurrence de banques soit absolument libre, libre d'émettre comme d' escompter $»^{25}$. Son argumentation est relativement radicale. Selon lui, la pluralité des banques doit favoriser un esprit de concurrence et faciliter la circulation d'une masse monétaire plus grande dans la mesure où les établissements escomptent du papier en masse ${ }^{26}$. Cette configuration plus favorable doit permettre une élévation du crédit et une augmentation des investissements. Enfin, il pense que la concurrence favorise la recherche de l'épargne.

${ }^{23}$ Smith (1990), p. 9.

24 Plessis (1985), p. 269.

25 Courcelle-Seneuil (1867), p. 38.

${ }^{26}$ Comme la plus grande partie des défenseurs de la banque libre, Jean-Gustave CourcelleSeneuil est partisan de la banking school (école de la banque) selon laquelle la monnaie de papier doit pouvoir être émise librement pour satisfaire la demande monétaire. Le principe de la convertibilité du billet oblige la banque à diminuer son émission quand l'encaisse est insuffisante. 
Après avoir montré tout l'intérêt de la concurrence, il examine les critiques faites à ce type d'organisation et tente d'établir une contre-argumentation. La critique principale repose sur l'émission exagérée de billets. Il considère cette argumentation comme spécieuse. La concurrence doit limiter de façon naturelle les émissions trop grandes. En effet, celles-ci se traduisent par des demandes de remboursement que les banques peuvent refuser en suspendant automatiquement leurs paiements.

L'auteur examine enfin la question de la garantie. Il admet que l'information des agents et la réputation des établissements sont des éléments importants dans le choix, mais il montre que la publicité du bilan doit permettre d'instaurer la confiance. Il refuse toute forme de réglementation sauf en matière de limitation de la fraude et de la contrefaçon. Dans une perspective similaire, Paul Coq s'inquiète du problème de l'unité de la monnaie et admet que faute d'équivalent l'échelle des prix s'en trouve modifiée. Il conteste l'idée des partisans du monopole qui affirment que l'Etat est garant de l'unité monétaire et répond que le seul rôle des pouvoirs publics est de battre monnaie et de certifier le poids de l'or. Il approuve l'idée de concurrence illimitée et démontre que celle-ci n'est pas incompatible avec l'unité monétaire dans la mesure où l'esprit marchand des banques suffit à instaurer la confiance ${ }^{27}$.

Très peu d'auteurs partagent les opinions radicales de Jean-Gustave Courcelle-Seneuil et de Paul Coq sur la liberté bancaire. John Horn considère, dans une perspective identique, que l'émission est une industrie privée et non une fonction ou une mission de service public ${ }^{28}$. La liberté équivaut à l'égalité, c'est-à-dire la liberté pour tous d'exercer cette industrie : "Nous voulons que la société qui s'établit pour faire des affaires de banque puisse à cet effet se servir de tous les moyens que les clients approuvent et qui ne sont réprouvées ni par la morale ni par la lo $i \gg^{29}$. Cette position refuse toute sorte de privilège bancaire.

Il estime également que le monopole est un système trompeur qui favorise plus les banqueroutes que la concurrence. Ainsi, selon John Horn, le modèle américain $^{30}$, pourtant décrié par les partisans du monopole, montre toute son effica-

${ }^{27}$ Coq (1865), p. 139 et suivantes

${ }^{28}$ Horn (1866), p. 366 et suivantes.

${ }^{29}$ Horn (1866), p. 392.

${ }^{30}$ Le modèle américain a toujours oscillé entre free banking et central banking. Depuis la Déclaration d'indépendance de 1776, les Etats indépendants peuvent créer un système bancaire autonome. Chaque banque a le monopole d'émission au sein d'un Etat. En 1791 une Banque des Etats-Unis est créée. Elle est financée par une dotation gouvernementale et possède un droit de contrôle sur la masse monétaire. La réforme de 1811 supprime cette banque et favorise la multiplication des établissements dans chaque Etat. Une nouvelle banque des Etats-Unis est fondée en 1816, elle reçoit une mission de prêteur en dernier ressort, mais n'a pas de monopole d'émission. Mais craignant que cette structure ne constitue une menace pour la liberté des citoyens, le Président Andrew Jackson en retire les fonds publics en 1833 et la supprime en 1836. De 1837 à 1863, le gouvernement décide d'alléger son contrôle. Dans les faits, la situation est complexe. Dans certains Etats (Michigan, New York, Georgie, etc.), l'organisation s'apparente au modèle de la banque libre. En revanche, dans les Etats du sud et de l'ouest, le monopole persiste. Le National Banking Act de 1863 instaure une monnaie unique et classe les établissements en deux grandes catégories. Les banques régionales (Country Banks) se spécialisent dans la gestion des dépôts et ont obligation de détenir un minimum de réserve placé dans une banque fédérale (national bank). Celles-ci peuvent émettre des billets sous réserve qu'ils soient entièrement gagés par des avoirs en fonds publics. Elles sont localisées dans quinze grandes villes. 
cité : de 1835 à 1860 , il n'y a pas eu d'excès d'émission et la circulation est passée de 103,7 millions à 207,1 millions de dollars. Robert Patterson complète l'analyse de John Horn et montre que la liberté d'émission favorise le contrôle d'une banque sur l'autre, notamment pour la question de la surémission des billets. La pluralité permet donc de freiner la hausse du taux d'intérêt et limite le danger relatif à l'excès d'émission des billets ${ }^{31}$.

La position des partisans de la libre concurrence est contestée tant par les économistes favorables au monopole que par ceux en accord avec la liberté d'émission. John Stuart Mill montre d'ailleurs, à la lumière du modèle américain, qu'une situation complètement concurrentielle se solderait tôt ou tard par l'établissement d'un oligopole $^{32}$. Il ne semble donc pas, selon lui, qu'il faille s'inquiéter du modèle de concurrence illimitée. L'intérêt du marché est qu'il se régule de façon automatique. Mais il admet que la pluralité entraînerait une facilité de crédit salutaire pour les uns et génératrice de spéculation pour les autres.

\section{La concurrence oligopolistique}

La situation oligopolistique dans le domaine bancaire rassemble la majorité des économistes favorables à la pluralité d'émission. Ceux-ci organisent leur argumentation autour de deux thèmes : le monopole d'émission ne constitue pas une solution souhaitable, mais la libre concurrence n'est pas non plus envisageable dans la mesure où elle présente trop de risques.

La critique du monopole se généralise. Comme le montre Emile de Laveleye : «Mais ce régime, condamné par l'opinion ne peut tarder à être modifié et remplacé... $»^{33}$. Ainsi, le monopole d'émission constituerait une infraction au droit commun dans la mesure où il ne s'appuie sur aucune réalité juridique ${ }^{34}$. Les économistes sont tous d'accord pour critiquer l'interventionnisme croissant de l'Etat, mais admettent la particularité du système bancaire et monétaire. La libre concurrence peut avoir des effets néfastes sur l'organisation économique. Antoine de Cherbuliez montre que «l'Etat possède certaines aptitudes qui manquent absolument à la société, et si la société lui est supérieure dans ce qu' elle peut comme lui, elle ne l'est certainement pas dans ce qu'elle ne peut d'aucune manière $»^{35}$.

Dans le domaine bancaire et monétaire, il semble donc important de conserver une part de l'interventionnisme étatique. L'Etat peut, en effet, assurer un service uniforme et offrir des garanties permanentes en fournissant à la circulation une monnaie unique et donc reconnue par l'ensemble des acteurs économiques. L'auteur montre que les modèles monétaires américains et écossais connaissent des abus et que la libre concurrence n'est pas totalement efficace. Ainsi, le contrôle par l'Etat de l'émission monétaire est un principe, selon Antoine de Cherbuliez, d'ordre public : «l'Etat doit faire de l'émission de billets au porteur un privilège

\footnotetext{
31 Patterson (1867), pp. 559-588.

32 Stuart Mill (1867), p. 593.

${ }^{33}$ Laveleye (1865), p. 186.

34 Ehrmann (1865).

${ }^{35}$ Cherbuliez (1867), p. 784.
} 
exclusif en faveur de certaines compagnies expressément autorisées ou même d'une seule $»^{36}$.

Léonce de Lavergne partage une analyse semblable selon laquelle : «la facilité de la circulation du billet de banque se proportionne à l'étendue de sa convertibilité ${ }^{37}$. Cette thèse repose sur l'information parfaite des acteurs : «le billet de banque est un simple effet de commerce qui inspire une plus grande confiance que les autres parce que les signatures sont plus connues et que la convertibilité est plus certaine $»^{38}$. Cette approche est fondamentale, l'auteur pense qu'une trop grande liberté entraînerait des désordres dans les signes qui conduirait de façon inéluctable à une crise monétaire. John Horn partage cette idé ${ }^{39}$, mais montre qu'elle ne se vérifie pas dans les pays où règne un modèle de banque libre, notamment en Ecosse.

Léonce de Lavergne est partisan d'une concurrence réglementée, il n'entend pas permettre l'établissement d'un nombre illimité d'établissements. L'exemple des banques départementales existantes avant 1848 constitue, selon lui, une bonne structure. Il propose même de partager le territoire en dix ou douze régions et de mettre en place « un nombre déterminé de banques instituées par la loi avec un privilège légal dans une circonscription déterminée $»^{40}$. Il propose la création d'une banque ayant le droit d'émission sur une zone déterminée et pour 100000 habitants ${ }^{41}$.

Son argumentation s'articule autour d'une critique des partisans du monopole. Il ne croit pas que la concurrence favorise un manque de sécurité dans la mesure où le système fonctionne autour de quelques compagnies reconnues et autorisées par la loi. Il admet explicitement que le billet présente plus de sûreté s'il est plus facilement reconnu et accepté. Mais si les banques concurrentes s'ouvrent réciproquement des crédits en remboursement de leurs billets, alors il ne devrait y avoir aucun problème. Ce dernier argument repose sur l'idée de solidarité, constituant la condition même du privilège. Léonce de Lavergne entend instituer une forme de solidarité qui permette aux banques d'inspirer une confiance au moins égale à celle de la Banque de France. Cette approche est partagée par Monsieur Cohen. Selon lui, la concurrence illimitée entre les banques favorise l'augmentation de l'escompte et est préjudiciable au commerce. Il paraît important de développer une solidarité entre les banques, une assistance mutuelle ${ }^{42}$.

La thèse de la solidarité des banques se développe progressivement dans les milieux économiques. John Horn montre que dans les pays où la concurrence est limitée, les billets des banques concurrentes sont acceptées, l'exemple de l'Ecosse est là pour le prouver. Il montre même, en s'appuyant sur les modèles suisses et prussiens, que les établissements peuvent s'entendre. En Prusse, une banque centrale supervise des banques provinciales concurrentes et facilite l'échange de

\footnotetext{
36 Cherbuliez (1867), p. 784.

${ }^{37}$ Lavergne (1867), p. 847.

${ }^{38}$ Lavergne (1867), p. 848.

39 Horn (1867), p. 580.

${ }^{40}$ Lavergne (1867), p. 850.

41 Lavergne (1865).

${ }^{42}$ Cohen (1867), p. 335.
} 
billets. En Suisse ${ }^{43}$, les banques ont fondé un cartel pour l'acceptation mutuelle de leurs billets ${ }^{44}$. En fait, il n'y a pas, selon Horn, d'inconvénients à la liberté sauf peut-être certains abus qui existent également dans le commerce.

\section{Le duopole : une solution favorable aux frères Péreire}

Les frères Péreire vont proposer à la commission une solution leur étant plus favorable : le duopole ${ }^{45}$. Cette structure permettrait une cohabitation sur l'ensemble du territoire entre les deux établissements bancaires ayant un droit d'émission : la Banque de France et la Banque de Savoie.

La thèse d'Isaac Péreire repose sur une critique du monopole et de la concurrence illimité ${ }^{46}$. La critique du monopole est essentiellement juridique : elle porte sur le privilège exclusif de la Banque de France sur l'ensemble du territoire. Pour Isaac Péreire, le monopole ne peut pas se justifier dans la mesure où aucune loi ne l'établit clairement ${ }^{47}$. Il y aurait donc, selon lui, une contradiction évidente entre la volonté des autorités et son application. Ainsi, l'Etat soutient le développement de l'esprit d'association, mais refuse la légalisation de la Banque de Savoie, qui pourtant va à l'encontre de la centralisation excessive du système bancaire. Il pense donc que l'ouverture du système à la concurrence limitée à deux établissements serait salutaire à l'esprit d'entreprise. Il ne demande pas pour autant la mise en œuvre d'une concurrence illimitée qui serait néfaste au bon fonctionnement de l'économie. Néanmoins, il pense que «les inconvénients du système de la pluralité ou de la liberté (sont) plus grands que ceux de l'unité ou du monopole ${ }^{48}$. D'ailleurs, il montre que le système de la pluralité expérimenté en Ecosse a plus d'avantages que d'inconvénients.

La pluralité, pour les frères Péreire, est certainement la solution la meilleure puisqu'elle permettrait de favoriser l'extension du commerce. Seule une forme particulière d'oligopole pourrait être satisfaisante : le duopole. Isaac Péreire montre que celui-ci permettrait de diminuer les inconvénients des deux systèmes, c'est-à-dire de conserver la confiance, tout en favorisant l'émergence d'une situation de concurrence. Cette solution consiste donc à organiser ce que des partisans d'une structure oligopolistique ont appelé la solidarité d'affaires : « ces banques se prêtent un mutuel appui et dans les temps de crise, elles ont conjuré efficacement par leur entente le danger des paniques ${ }^{49}$.

${ }^{43}$ Le système bancaire suisse s'est constitué sur le modèle du free banking. Au début du $\mathrm{XIX}^{\mathrm{e}}$ siècle, les Etats de la Confédération autorisent l'émission de billets pour les établissements privés. Les banques s'échangent mutuellement leurs billets. Le marché bancaire est de nature oligopolistique. Aucun établissement ne domine le marché et le fonctionnement du système se caractérise par une absence de perturbation.

${ }^{44}$ Horn (1867), p. 580.

${ }^{45}$ Le duopole est une situation de marché, une forme particulière de l'oligopole où l'offre est le fruit de deux entreprises seulement.

${ }^{46}$ Péreire, Isaac (1864a).

47 Péreire, Isaac (1864b).

48 Péreire, Isaac (1865).

${ }^{49}$ Péreire, Isaac (1865), p. 142. 
La solidarité d'affaires conduirait, selon Emile Péreire, à une situation économique favorable pour le commerce et l'industrie dans la mesure où elle facilite l'élargissement des moyens de crédit ${ }^{50}$. Monsieur Hubert-Delisle n'est pas d'accord avec cette approche. Il se demande, très justement, comment la solidarité d'affaires est-elle possible entre deux concurrents et estime que, à terme, elle conduirait à un épuisement de l'encaisse de la Banque de France en raison des différences d'escompte entre les deux établissements. Isaac Péreire conteste ce point de vue et s'appuie sur le modèle anglais pour étayer sa contre-argumentation : en séparant les départements de l'escompte et de l'émission et en limitant l'émission de billet, le problème doit être résolu.

\section{L'acceptation du monopole d'émission et ses implications économiques}

Face aux conceptions diverses et opposées de la pluralité d'émission, les partisans du monopole présentent plus d'unité. Celui-ci s’intègre plus généralement dans un questionnement sur l'intervention de l'Etat dans l'économie.

\section{Légitimation théorique du monopole d'émission}

Walter Bagehot ouvre les débats de la commission d'enquête. Il est plutôt favorable au principe de la banque centrale, mais reconnaît que le modèle de la banque libre présente l'avantage de développer rapidement l'activité et les innovations bancaires dans un pays neuf. Il vaut donc mieux qu'il y ait une forme de pluralité : «la faculté d'émission accordée à un certain nombre de banques a pour résultat de développer les dépôts et de multiplier l'ensemble des opérations de banques $»^{51}$. Malgré cette conception, il reste favorable au principe de la banque centrale et montre que le système anglais présente certains intérêts dans la mesure où la concurrence est respectée sous le contrôle de la Banque d'Angleterre.

Dans cette perspective, il va développer la théorie du prêteur en dernier ressort $^{52}$. Ce rôle appartient à la banque centrale, il permet de suppléer aux problèmes de liquidité et doit s'exercer dans la transparence. Il comporte trois règles de fonctionnement : la banque centrale doit avoir un rôle de soutien, impose des taux d'intérêt de pénalisation aux établissements qui demandent son aide et doit exclure les banques insolvables de l'accès au réescompte. La banque centrale a donc un rôle important dans la mesure où elle doit assurer l'unité du billet et éliminer les institutions insolvables ${ }^{53}$.

Louis Wolowski est également un fervent partisan du système de Banque centrale. Il montre que l'émission est le cœur du système économique et tout en se réclamant des économistes classiques, il justifie et reconnaît « le devoir de l'Etat d'intervenir, de surveiller, de limiter l'exercice de la faculté d'émission ${ }^{54}$. Son

\footnotetext{
${ }^{50}$ Péreire, Emile (1866).

51 Bagehot (1867).

${ }^{52}$ La thèse du prêteur en dernier ressort a été développée au XIX ${ }^{\mathrm{e}}$ siècle par Thornton (prêteur ultime). Diatkine (1998), pp. 201-230.

53 Bagehot (1962).

54 Wolowski (1867), p. 187.
} 
argumentation repose avant tout sur la confusion des thèses en faveur du libéralisme bancaire. En effet, certaines admettent la nécessité de fixer une limite plus ou moins grande aux créations bancaires et recherchent des garanties contre les manœuvres frauduleuses. Victor Couillet partage cette opinion et craint que la concurrence entraîne des abus.

Il paraît donc nécessaire de protéger la population contre d'éventuels problèmes. Cette fonction publique s'apparente donc à un droit régalien. De façon plus générale, Louis Wolowski admet que la pluralité risque d'accroître le volume de la masse monétaire en circulation. Il partage les craintes de Clément Juglar qui a montré que l'excès d'émission favorise les crises économiques ${ }^{55}$. Dans une perspective de currency school, François Ducuing montre que le billet ne peut correspondre qu'à une transaction commerciale consommée. Il critique ainsi la thèse des partisans de la pluralité d'émission et n'admet pas qu'un billet soit analysé comme un découvert de circulation ${ }^{56}$.

La crainte plus grande des partisans du monopole repose sur la rupture de l'unité bancaire. Cette crainte est d'ailleurs, comme nous l'avons vu, partagée par des économistes favorables à une structure oligopolistique. Le monopole d'émission doit favoriser la circulation de l'information bancaire. Sans celle-ci, les agents ne sont plus informés et le billet n'a donc plus la même valeur. Ainsi, le monopole permet de garantir l'unité de la monnaie en donnant à tous un accès égal. Michel Chevalier n'est pas insensible à ce type d'argumentation. Il admet que la circulation d'une monnaie viciée soit contraire à l'ordre public, mais il montre, à la suite de Léonce de Lavergne, que l'organisation concurrentielle n'est pas contraire à la qualité de la monnaie dans la mesure où les établissements peuvent s'entendre sur la qualité des billets à échanger.

Enrico Cernuschi renvoie dos à dos monopole d'émission et pluralité dans la mesure où ces deux formes ne peuvent pas donner d'argent bon marché quand il n'y en a pas ou quand il est cher. Il refuse le monopole d'émission : «je refuse la loi en matière d'échange $»^{57}$, mais il montre également que l'introduction d'un système de liberté serait la cause de la disparition du billet. Néanmoins, il pense que l'adoption d'une règle en matière monétaire exige la constitution de réserves importantes. Le modèle de banque libre permet, selon lui, d'atteindre assez rapidement ce résultat. Il conclue en montrant que le meilleur système est le sien : pas de banque d'émission. Sa thèse repose sur la fin du billet de banque. Il propose que le billet corresponde dorénavant à une quantité de métal précieux déposée ${ }^{58}$.

\section{La confirmation du monopole d'émission}

Le Conseil supérieur, pour les besoins de l'enquête, a entendu 77 spécialistes et a reçu les réponses écrites de 27 personnes en 29 séances du 7 février 1865 au 20 juillet 1866. Dans son rapport, Monsieur de Lavenay montre qu'une grande

\footnotetext{
55 Juglar (1868).

56 Ducuing (1867), pp. 141 et s.

57 Cernuschi (1867), p. 317.

${ }^{58}$ Contrairement à l'ensemble des partisans de la banque libre, E. Cernuschi est partisan de la currency school.
} 
majorité se dégage en faveur d'un «grand établissement privilégié et surveillé par l'Etat, mais distinct et indépendant de l'Etat $»^{59}$. Mais cette position est loin de faire l'unanimité. Michel Chevalier pense que la banque est une industrie comme une autre, elle mérite donc, comme toute activité économique, d'être organisée sur un marché concurrentiel. La liberté des banques est le corollaire de la liberté du travail et de la liberté de l'industrie.

Suite aux propos de Michel Chevalier, le Président de la Commission, Eugène Rouher, met la question suivante au vote : «Y a-t-il lieu de confier l'émission de la monnaie fiduciaire à une banque unique et privilégiée ? » Le Conseil supérieur se prononce massivement en faveur du monopole d'émission. Il est vrai que l'ensemble des participants se sont, au cours de l'enquête, prononcés pour celui-ci : sur 191 auditions, 122 préfèrent la banque centrale, 27 la banque libre et 42 ne se prononcent pas ${ }^{60}$.

De façon générale, il faut replacer ce débat dans sa perspective historique. En effet, cette période se traduit pas un questionnement sur l'intervention de l'Etat. Ce dernier doit avoir un rôle essentiel dans l'activité économique, il doit « construire et éblouir à la fois » ${ }^{61}$. L'empereur a une conception particulièrement interventionniste de l'Etat. Celui-ci doit avoir un rôle stimulateur dans l'activité économique soit en l'organisant lui-même (politique de grands travaux) soit en la subventionnant. On ne parle pas encore de nationalisations, mais bon nombre d'économistes affirment la fonction prépondérante de l'Etat dans la relance et la transformation des structures économiques. Il peut le faire de façon directe en orientant ses dépenses, mais cette technique suscite l'opposition des partisans de l'orthodoxie budgétaire ${ }^{62}$.

La croissance économique et la nécessité de moderniser le système productif reposent donc sur une transformation radicale des moyens de financement. La Banque de France augmente son capital de 91 à 182 millions de francs et voit ses opérations d'escompte augmenter de façon considérable. A celles-ci s'ajoutent de nouveaux types d'opérations : les avances aux entreprises sur leurs actions et obligations, les prêts directs. La Banque de France devient donc le pivot du système de crédit. L'Etat va donc exercer une tutelle sur des institutions-relais (Banque de France, Caisse des dépôts, etc.), soit en nommant leur dirigeants, soit en conservant un droit de regard sur la comptabilité ${ }^{63}$.

On comprend donc tout l'intérêt du débat. La confirmation du monopole d'émission de la Banque de France s'inscrit dans une logique interventionniste. Il s'agit de donner à l'Etat un instrument qui lui permette d'orienter de façon efficace la politique monétaire de façon dynamique. La banque centrale est donc appréhendée comme un moyen de développement industriel.

\footnotetext{
59 Lavenay (1867), p. 25.

${ }^{60}$ Récapitulation des opinions des déposants (1865-1866), Archives de la Banque de France, DC $34 / 2888$.

${ }^{61}$ Bouvier (1967).

62 Plessis (1984), pp. 86-87.

${ }^{63}$ Allinne, Lescure (1981), pp. 280-293.
} 


\section{CONCLUSION}

Suite à la confirmation du monopole d'émission de la Banque de France, le débat entre free et central Banking va perdurer, mais progressivement se déplacer vers la question importante du bimétallisme jusqu'à son abandon en 1878.

Les attaques successives des économistes libéraux, des commerçants et des administrateurs du Crédit mobilier n'auront pas réussi à déstabiliser la Banque de France. En effet, celle-ci dispose d'appuis solides dans les milieux gouvernementaux, notamment d'Achille Fould, et sa politique de collaboration avec l'Etat et les grandes compagnies ferroviaires renforce sa position institutionnelle ${ }^{64}$.

La confirmation du monopole d'émission s'inscrit plus généralement dans la perspective d'une transformation monétaire. L'Etat va alors accélérer la transformation du dispositif bancaire. La logique est double. L'Etat entend, dans un premier temps, favoriser le financement des investissements grâce au développement des établissements privés. Les pouvoirs publics veulent également rétablir l'ordre financier autour de la Banque de France.

\section{SOURCES \\ ET BIBLIOGRAPHIE}

Aftalion, Florin, L'économie de la Révolution française, Paris, Hachette Pluriel, 1987.

Aglietta, Michel, «Genèse des banques centrales et la légitimité de la monnaie », Annales ESC, n 3 , mai-juin 1992, pp. 675-698.

Allinne, Jean-Pierre, Lescure, Michel, «Pour une étude des appareils économiques d'Etat en France au XIX ${ }^{\mathrm{e}}$ siècle », Annales ESC, mars 1981, pp. 280-293.

Asselain, Jean-Charles, Histoire économique de la France du XVIII siècle à nos jours, Tome I, Paris, Editions du Seuil, 1984.

Aubin, Christian, «Les assignats sous la Révolution française : un exemple d'hyperinflation », Revue économique, vol. 42, n 4, 1991, pp. 745-762.

Autin, Jean, Les frères Péreire, le bonheur d' entreprendre, Paris, Perrin, 1984.

Bagehot, Walter, «Audition du 17 février 1865 », in: Enquête sur les principes et les faits généraux qui régissent la circulation monétaire et fiduciaire, Tome I, Paris, Imprimerie impériale, 1867, pp. 18-56.

Bagehot, Walter, Lombard Street : a description of the money market (1873), London, reprint Homewood, 1962.

Bonnet, Victor, La liberté des banques d'émission et le taux de l'intérêt, Paris, éditions Guillaumin, 1864.

Bergeron, Louis, Les Rothschild et les autres, la gloire des banquiers, Paris, Perrin, 1991.

Bouvier, Jean, Les Rothschild, Paris, Fayard, 1967.

Bouvier, Jean, Les Rothschild, histoire d' un capitalisme familial, Bruxelles, éditions complexes, 1992.

Cameron, Rondo, Banking in the early stages of industrialization. A study in comparative economic history, New York, Oxford University Press, 1967.

Cernuschi, Enrico, « Audition du 24 octobre 1865 », in : Enquête sur les principes et les faits généraux qui régissent la circulation monétaire et fiduciaire, Tome I, Paris, Imprimerie impériale, 1867, pp. 296-332.

\footnotetext{
${ }^{64}$ Plessis (1985), pp. 270-271.
} 
Cherbuliez, de, Antoine, «Déposition écrite », in : Enquête sur les principes et les faits généraux qui régissent la circulation monétaire et fiduciaire, Tome V, Paris, Imprimerie impériale, 1867, pp. 769-784.

Cohen, Joseph, « Audition du 25 novembre 1865 », in: Enquête sur les principes et les faits généraux qui régissent la circulation monétaire et fiduciaire, Tome II, Paris, Imprimerie impériale, 1867, pp. 300-347.

Commez, Auguste, Question des banques, Banque de France-Banque de Savoie, Imprimerie Troyes ouvriers réunis, 1864.

Coq, Paul, Les circulations en banque ou l'impasse du monopole, Paris, éditions Guillaumin, 1865.

Courcelle-Seneuil, Jean-Gustave, « Audition du 16 décembre 1865 », in : Enquête sur les principes et les faits généraux qui régissent la circulation monétaire et fiduciaire, Tome III, Paris, Imprimerie impériale, 1867, pp. 1-64.

Dagneaux, A., Le crédit libre, par le travail libre et le Saint-Simonisme à l'auvre, Paris, éditions Dentu, 1864.

Diatkine, Sylvie, «Banque centrale et structure du système bancaire : les enseignements des débats monétaires anglais au début du XIX ${ }^{\mathrm{e}}$ siècle », Revue Française d'Economie, vol. 13, n 2, 1998, pp. 201-230.

Ducuing, François, « Audition du 18 novembre 1865 », in : Enquête sur les principes et les faits généraux qui régissent la circulation monétaire et fiduciaire, Tome II, Paris, Imprimerie impériale, 1867, pp. 141-166.

Ehrmann, Edmond, Considérations sur la cherté de l'argent, sur la part de responsabilité qui en revient à la Banque de France et sur mes moyens d'y remédier, Strasbourg, éditions Heitz, 1865.

Furet, Théophile, La Banque de France et la Banque de Savoie ou réduction et fixité du taux de l'escompte des banques privilégiées, Paris, éditions A. Blayes, 1864.

Horn, John, « Audition du 4 novembre 1865 », in : Enquête sur les principes et les faits généraux qui régissent la circulation monétaire et fiduciaire, Tome I, Paris, Imprimerie impériale, 1867, pp. 551-600.

Horn, John, La liberté des banques, Paris, éditions Guillaumin, 1866.

Juglar, Clément, Du change et de la liberté d'émission, Paris, éditions Guillaumin, 1868.

Laveleye, de, Emile, Le marché monétaire et ses crises depuis cinquante ans, Paris, éditions Guillau$\min , 1865$.

Lavenay, «Rapport présenté au Conseil supérieur du commerce, de l'agriculture et de l'industrie », in : Enquête sur les principes et les faits généraux qui régissent la circulation monétaire et fiduciaire, Tome VI, Paris, Imprimerie impériale, 1867.

Lavergne, de, Léonce, La Banque de France et les banques départementales, Paris, éditions Guillau$\min , 1865$.

Lavergne, de, Léonce, « Audition du 12 décembre 1865 », in : Enquête sur les principes et les faits généraux qui régissent la circulation monétaire et fiduciaire, Tome II, Paris, Imprimerie impériale, 1867, pp. 843-890.

Mill John Stuart « Audition », in: Enquête sur les principes et les faits généraux qui régissent la circulation monétaire et fiduciaire, Tome V, Paris, Imprimerie impériale, 1867, pp. 589-596.

Patterson, Robert H., «Audition », in: Enquête sur les principes et les faits généraux qui régissent la circulation monétaire et fiduciaire, Tome V, Paris, Imprimerie impériale, 1867, pp. 559-588.

Péreire, Isaac, La Banque de France et l'organisation du crédit en France, Paris, Imprimerie Paul Dupont, 1864a.

Péreire, Isaac, Réorganisation des Banques, légalité et urgence d' une réforme, Paris, Imprimerie Paul Dupont, 1864b.

Péreire, Emile, Déposition devant le Conseil supérieur du commerce (séance du 7 novembre 1865), Paris, Imprimerie Paul Dupont, 1866.

Péreire, Isaac, Déposition devant le Conseil supérieur du commerce (séance du 26 décembre 1865), Paris, Imprimerie Paul Dupont, 1866. 
Plessis, Alain, De la fête impériale au mur des fédérés (1852-1871), Paris, éditions du Seuil, 1976. Plessis, Alain, La politique de la Banque de France de 1851 à 1870, Genève, Droz, 1985.

Reyre, Clément, De la Banque de France et spécialement des variations du taux de ses escomptes, Lyon, Imprimerie Nigon, 1864.

Smith, Vera, The rationale of central banking and the free banking alternative (1936), Indianapolis, Liberty Press, 1990.

Valdenaire, M., Des banques privilégiées, bas prix et fixité de l'escompte, Paris, Imprimerie Paul Dupont, 1864.

White E., «Free Banking during the French Revolution », Exploration in Economic History, vol. 27, 1987, pp. 251-276

Wolowski, Louis, « Audition du 22 novembre 1865 », in: Enquête sur les principes et les faits généraux qui régissent la circulation monétaire et fiduciaire, Tome II, Paris, Imprimerie impériale, 1867, pp. 167-245. 